\title{
Hematoma Retroesofágico com Grave Disfagia após Estreptoquinase para Tratamento da Trombose Venosa Axilo-Subclávia
}

\author{
A ntonio Claudio do Amaral Baruzzi, Maria Emília Cardoso Gadelha, Claudio Cirenza, Elias Knobel \\ São Paulo, SP
}

Homem de 72 anos, portador de neoplasia de próstata estádio IV, submetido à osteossíntese da coluna cervical para descompressão metastática da raiz nervosa, responsável por dor intensa no membro superior direito. Após três meses da internação, apresentou trombose total da veia axilo-subclávia direita, complicação da cateterização prolongada da veia subclávia direita para controle de septicemia secundária a pneumonia hospitalar.

Foi submetido a terapia trombolítica com estreptoquinase por via venosa, no membro superior contra-lateral, na dose de 250.000UI em 15min, seguida de 100.000UI/h durante cinco dias, com total recanalização do trombo e redução do edema. Após 24 h do término da trombólise, apresentou disfagia a líquidos e o esofagograma mostrou extensa compressão extrínseca do esôfago, por provável hematoma retroesofágico. Necessitou de nutrição enteral durante três meses, quando ocorreu normalização da de glutição, reabsorção do hematoma retroesofágico e passagem adequada do contraste pelo esôfago. No seguimento evolutivo não apresentou seqüelas da trombose venosa profunda, não tem disfagia e permaneceu em uso profilático de meias elásticas e heparina de baixo peso molecular.

\section{Severe Dysfagia Due to Retroesophageal Hematoma after Thrombolytic Therapy for Right Axillosubclavian Vein Thrombosis}

We report the case of a 72 year-old man with advanced, stage IV, prostate cancer who underwent osteosynthesis of the cervical spine for nerve root decompression due to metastasis which was causing severe pain in his right upper limb. After three months in the hospital, he developed occlusive thrombosis of the right axillosubclavian vein as a complication of prolonged catheterization of the right subclavian vein for treatment of septicemia secondary to a hospital acquired pneumonia.

The patient received thrombolytic therapy with IV streptokinase in the contralateral arm in the following dosage: 250,000 units in 15 minutes followed by 100,000 units per hour during five days. This led to total recanalization of the thrombus, with significant reduction of the arm edema. Twenty-four hours after the end of the thrombolytic therapy, the patient started to complain of dysfagia to solids and liquids and a contrasted esophagogram revealed extensive extrinsic compression of the esophagus due to a probable retroesophageal hematoma. The patient required enteral nutrition via nasoenteral tube during three months after which swallowing returned to normal and a repeat upper GI series confirmed that the hematoma had been reabsorbed, with normal passage of contrast through the esophagus. On late follow-up, the patient did not show evidence of any sequelae of deep venous thrombosis nor any residual dysfagia and is currently in use of elastic stockings and low molecular weight heparin.

Arq Bras Cardiol, volume 69 (n' 2), 125-127, 1997

Hospital Israelita Albert Einstein - São Paulo

Correspondência: Antonio Claudio A. Baruzzi - Hospital Israelita Albert Einstein - CTI - Av. Albert Einstein, 627 - 5 - 05651-901 - São Paulo, SP

Recebido para publicação em 29/4/97

Aceito em 19/6/97
Nos últimos anos, os trombolíticos têm sido utilizados em outras situações clínicas além do infarto agudo do miocárdio (IAM), por exemplo, no acidente vascular cerebral isquêmico agudo, no tromboembolismo pulmonar, na trombose venosa profunda (TVP), nas obstruções arteriais 
trombóticas e embólicas, nas obstruções de cateteres vasculares e na trombose da artéria e veia central da retina ${ }^{1}$.

Entretanto, não é terapêutica isenta de riscos, sendo a complicação mais temida os eventos hemorrágicos relacionados, principalmente, ao sistema nervoso central e em locais onde a compressão extrínseca do vaso relacionado ao sangramento não possa ser realizada.

Relatamos caso de disfagia por compressão extrínseca do esôfago devida a hematoma retroesofágico após uso de estreptoquinase (SQ) para tratamento de trombose venosa axilo-subclávia, em portador de neoplasia de próstata.

\section{Relato do Caso}

Homem de 72 anos, portador de neoplasia de próstata estadio IV, submetido a osteossíntese da coluna cervical com prótese metálica para descompressão metastática da raiz nervosa, responsável por dor intensa no membro superior direito. $\mathrm{No}^{\circ}$ dia do pós-operatório foi transferido para este Hospital, onde necessitou de traqueostomia, devida à paralisia da corda vocal direita e paresia da esquerda (provável trauma cirúrgico do nervo laríngeo-recorrente), cateterização prolongada da veia subclávia direita para antibioticoterapia, nutrição parenteral, drogas vasoativas e monitorização hemodinâmica com o cateter de Swan-Ganz para controle de septicemia secundária a pneumonia hospitalar.

Após três meses da internação, desenvolveu importante edema do membro superior direito com restrição funcional de sua movimentação. O estudo vascular com ultrasom constatou trombose total da veia axilo-subclávia direita, sem comprometimento do sistema arterial.

Foi submetido a terapia trombolítica com SQ, por via venosa no membro superior contra-lateral, na dose de $250.000 \mathrm{UI}$ em $15 \mathrm{~min}$ seguida de $100.000 \mathrm{UI} / \mathrm{h}$ durante cinco dias, período necessário para que houvesse total recanalização do trombo, conforme seguimento evolutivo diário com a ultra-sonografia vascular. Vinte e quatro horas após o término da trombólise, queixou-se de disfagia a líquidos. $\mathrm{O}$ estudo contrastado do esôfago mostrou extensa compressão extrínseca do mesmo, por provável hematoma retroesofágico (fig. 1A e 1B). Apesar de não ter ocorrido queda do hematócrito e o fibrinogênio sérico situar-se acima de $100 \mathrm{mg} \%$, foram utilizadas duas unidades de plasma fresco para normalizar as discretas alterações da coagulação intrínseca (atividade protrombínica de $45 \%$ ) e extrínseca (tempo de tromboplastina parcial ativada de $65 \mathrm{~s}$ ). A contagem de plaquetas era normal.

Devido à gravidade da disfagia, foi posicionada sonda para dieta enteral; somente após três meses ocorreu normalização da deglutição, constando-se reabsorção do hematoma retroesofágico e passagem adequada do contraste pelo esôfago (fig. 1C). No seguimento evolutivo de seis meses após a cirurgia, houve também retorno dos movimentos das cordas vocais, confirmando a hipótese clínica de lesão não definitiva do nervo laríngeo-recorrente. Presentemente, encontra-se sem deficiência funcional do membro superior direito, sem disfagia e em programa de fechamento ambulatorial da traqueostomia.

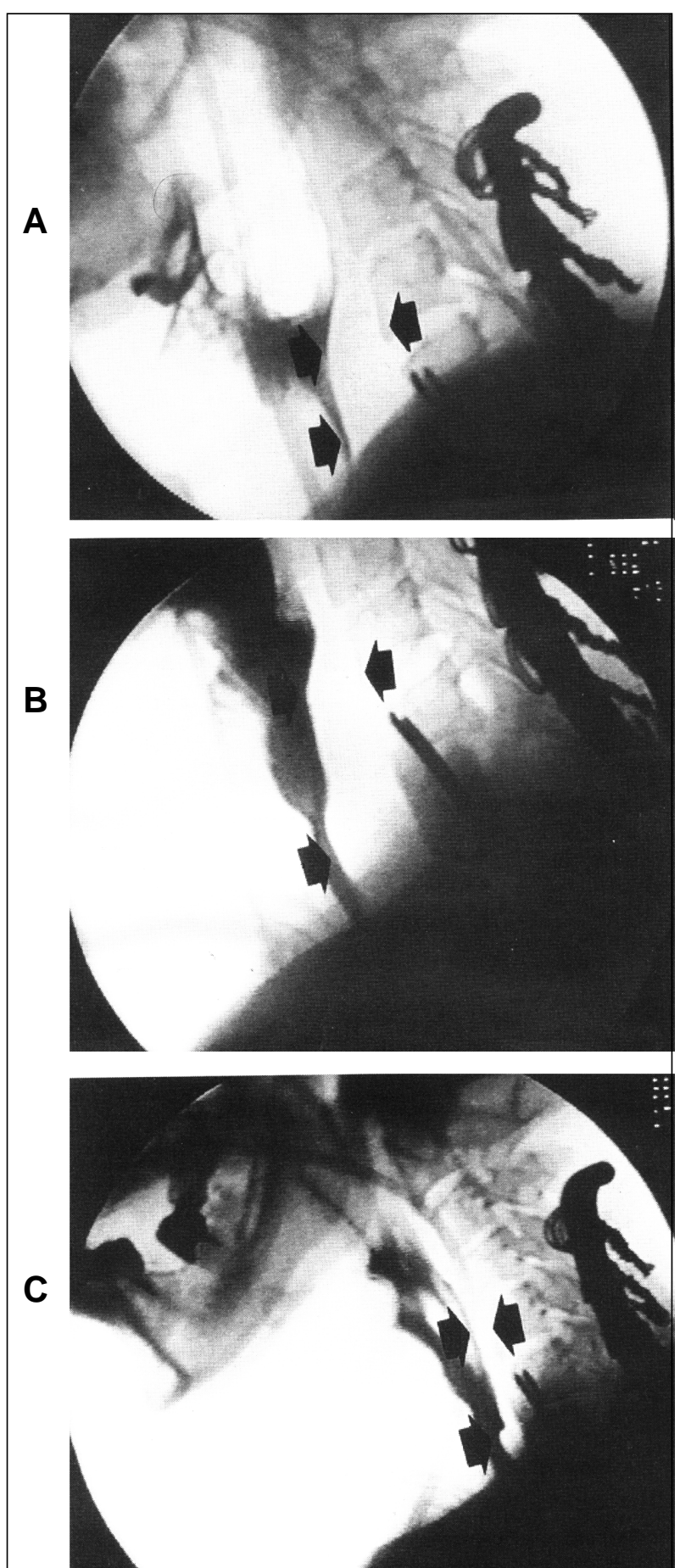

Fig. 1 - A e B) Grave compressão extrínseca do esôfago secundário a extenso hematoma retreoesofágico (área delimitada pelas setas); C) controle evolutivo após 3 meses. Reabsorção do hematoma com espaço retroesofágico (setas) normal.

\section{Discussão}

O uso de trombolíticos para o tratamento da TVP tem sido motivo de várias publicações ${ }^{2}$, inclusive as relacionadas à trombose venosa axilo-subclávia. Entretanto, as doses e a via de administração, seja através de cateterização venosa seletiva com injeção do trombolítico junto ao trombo, 
ou por via venosa sistêmica, não estão bem definidas. Rogers e col $^{3}$ advogam seu uso nos pacientes com história de até 14 dias de TVP, na dose descrita neste caso, durante três a cinco dias, até que se demonstre a recanalização do trombo por exame não-invasivo (ultra-som vascular) ou pela venografia. Pilger e $\mathrm{col}^{4}$ recomendam doses elevadas por curto período de tempo, por exemplo, seis milhões de unidades de SQ durante 4 h. Não existe demonstração quanto à superioridade de um trombolítico sobre os demais para o tratamento da TVP.

Várias complicações hemorrágicas têm sido descritas com o uso de trombolíticos, como hematomas de língua, subcapsular do fígado, de face, de mama, de tecidos moles nos locais de punção vascular e até fatais, como nos sangramentos do sistema nervoso central. Para o controle do sangramento deve ser utilizado criopreciptado (fibrinogênio abaixo de $100 \mathrm{mg} \%$ ), plasma fresco (reposição de fatores da coagulação e de inibidores do plasminogênio tecidual), plaquetas e de concentrado de hemácias, quando houver queda da hemoglobina $>2 \mathrm{~g}$.

O sangramento retroesofágico secundário ao trombolítico é um evento muito raro e não encontramos referência na literatura, exceto dissecção da mucosa do esôfago ${ }^{5}$ após o seu uso no tratamento do IAM. Neste paciente, houve nítida relação entre o início da disfagia e o uso do trombolítico. Embora o trombolítico tenha sido utilizado após três meses da osteossíntese da coluna cervical, é provável que o hematoma tenha ocorrido em área cruenta, sob cicatrização incompleta. Devido à ausência de alterações hemodinâmicas, opta- mos por observar sua evolução, uma vez que qualquer abordagem cirúrgica descompressiva poderia resultar em complicações também graves, como mediastinite, fístulas pela deficiência nutricional e outras.

O controle evolutivo a longo prazo de portadores de TVP tem demonstrado significativa incidência de síndrome pós-TVP, ao contrário daqueles submetidos à terapia trombolítica. Por este motivo, várias publicações a têm recomendado como terapêutica inicial da TVP, seguida da heparinização sistêmica.

A reabsorção do hematoma retroesofágico, com conseqüente normalização da deglutição e retorno da mobilidade das cordas vocais, permitindo o fechamento da traqueostomia, foram fatores clínicos importantes que contribuíram para evolução satisfatória. Decorridos seis meses do evento, o paciente encontra-se em tratamento hormonal antiandrogênico para a neoplasia da próstata, porém sem anticoagulação oral profilática pelas dificuldades de controle laboratorial. Devido ao risco de recorrência de TVP, especialmente por ser portador de neoplasia, está utilizando meias elásticas de compressão média e heparina de baixo peso molecular ${ }^{6}$.

Esta rara complicação hemorrágica foi importante sinal de alerta, pois os eventos hemorrágicos ocorrem de forma inesperada, por vezes fatal. É importante a contínua vigilância médica nos pacientes sob tal tratamento trombolítico, bem como o esclarecimento dos familiares e do paciente quanto aos riscos inerentes ao seu uso, independente de sua indicação.

\section{Referências}

1. Comerota AL - Thrombolytic Therapy. Philadelphia: JB Lippincott, 1995: 525.

2. Comerota AJ, Katz ML, White JV - Thrombolytic therapy for deep venous thrombosis: how much is enough? Cardiovasc Surg 1996; 4: 101-1.

3. Rogers LQ, Lutcher CL - Streptokinase therapy for deep vein thrombosis: A comprehensive review of the English literature. Am J Med 1990; 88: 389-95.

4. Pilger E, Eriksson H, Grungreiff K et al - Multicenter studies of ultra-high dose, short-duration streptokinase treatment of deep vein thrombosis. Curr Therap Res 1996; 57: 251-66.

5. Jishi F, Sissons CE, Silverstone EJ et al - Oesophageal dissections after thrombolytic treatment for myocardial infarction. Torax 1992; 47: 835-6.

6. Baruzzi ACA, Nussbacher A, Lagudis S, Marcondes JA - Trombose venosa profunda: Profilaxia. Arq Bras Cardiol 1996; 67:17-20. 